\title{
Johannes Leo Africanus alias al-Hassan al-Wazzan
}

Mit der transatlantischen Expansion der Iberischen Halbinsel rückte auch die afrikanische Gegenküste Spaniens seit der Endphase der Reconquista und dem Beginn der amerikanischen Conquista wieder zunehmend in die Reichweite abendländischer Ausdehnungsgelüste. Auch wenn die Reconquista buchstäblich in die Conquista umschlug, sie fast vollständig absorbierte und damit alle Expansionsbewegungen nach Westen auf die Neue Welt umgelenkt wurden, waren sich doch viele der iberischen Autoren der Möglichkeiten sehr wohl bewusst, die relativ schwach verteidigte Gegenküste des afrikanischen Kontinents zu attackieren.

Doch den Europäern - und insbesondere den Spaniern - wurde zunehmend klar, wie wenig man von der arabischen Gegenküste und vor allem vom Inneren des afrikanischen Kontinents wusste. Afrika war für die Europäer noch immer eine weitestgehend unbekannte Welt, die inneren Differenzierungen dieser Welt waren ein großer weißer Fleck auf Europas Kartenwerken. Dies begann sich mit dem wichtigsten und bis hinein ins 19. Jahrhundert hinein einflussreichsten Autor des 16. Jahrhunderts zu ändern, der mit seiner Beschreibung Afrikas ein Standardwerk gerade für die europäischen Leserschaften schuf, welchem ein überaus langes Fortleben beschieden sein sollte. Bereits die Geschichte dieses Schriftstellers ist überaus aufschlussreich und verweist auf jene historischen Konstellationen, mit denen wir uns zu Beginn dieses Teils unserer Vorlesung beschäftigt haben. Doch stellen wir sie noch einen Augenblick zurück.

An der Wende zum 16. Jahrhundert befinden wir uns zweifellos in einer Epoche, in der man zunehmend lernte, die Welt, in der man lebte, weltumspannend zu denken und Weltregionen miteinzubeziehen, an die man zuvor niemals gedacht hätte. Dies galt in politischen, ökonomischen und militärischen Dingen, aber auch in jenen, die wir mit Michel Foucault als biopolitisch bezeichnen dürfen. Denn mit dem Aufbau wirtschaftlicher Strukturen wie etwa der Plantagenwirtschaft oder dem forcierten Bergbau wurde es notwendig, Arbeitskräfte zu beschaffen, die vor Ort nur in beschränktem Umfang zur Verfügung standen. Hier suchte das frühkapitalistische System, das sich in einem zunehmend weltumspannenden Rahmen formierte und definierte, nach Abhilfe und nach biopolitischen Lösungen, die weltweiten Zuschnitts waren.

Auch der spanische Dominikanermönch Fray Bartolomé de Las Casas, auf den wir schon beim Bordbuch des Columbus gestoßen waren, dachte im weltweiten Maßstab und versuchte, die Welt der neuen Entdeckungen in sein christliches Weltbild zu integrieren. Er trat als einer der Beschützer der unterdrückten, versklavten, zunehmend vernichteten Ureinwohner Amerikas, der indigenen 
Bevölkerung, auf und kämpfte lange Jahrzehnte auf allen erdenklichen Ebenen gegen die gewalttätige Ausbeutung der Indios durch jene, die sie eigentlich zum wahren Glauben hätten führen sollen. Zugleich befürwortete er die Ersetzung der indigenen Arbeitskraft durch aus Afrika eingeführte schwarze Sklaven, die auf den Plantagen wie auch im Bergbau eingesetzt werden konnten und über eine größere Widerstandsfähigkeit und längere Lebensdauer verfügten als die indianische Bevölkerung. Sie verstehen, dass derlei Überlegungen sofortige biopolitische Konsequenzen nach sich ziehen mussten, waren damit doch Deportationen und Umsiedelungen verbunden, die den afrikanischen Kontinent in den transatlantischen Handel miteinbezogen und letztlich das heraufführten, was man im Zeichen brutalster Sklaverei und Ausbeutung mit Recht als den Black Atlantic bezeichnet hat. ${ }^{1}$

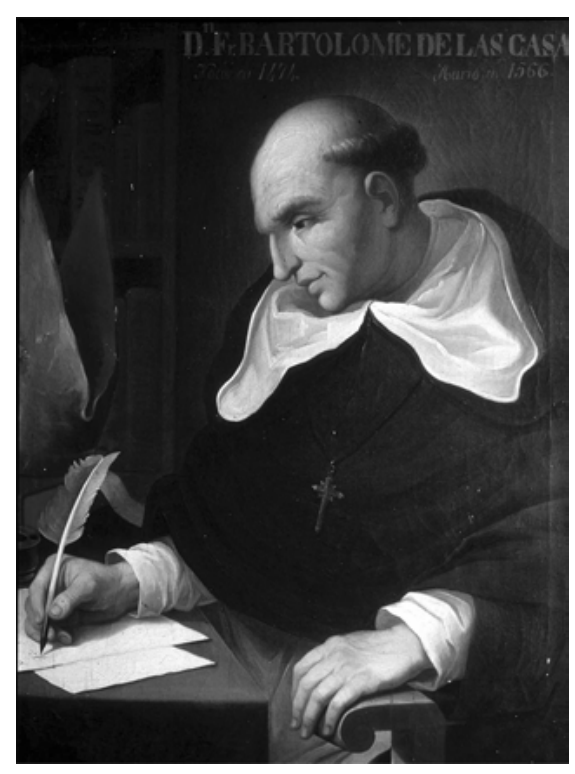

Abb. 47: Bartolomé de las Casas (Sevilla, 1484 oder 1485 - Madrid, 1566).

Es ist hier weder der Ort noch haben wir die Zeit, um uns mit dem Lebenswerk von Bartolomé de Las Casas tiefergehend auseinanderzusetzen. Doch die Bedeutung dieses Dominikanermönches innerhalb der in dieser Vorlesung skizzierten welthistorischen Entwicklungen kann kaum überschätzt werden, führte er doch auch eine Reihe von Argumenten etwa der Menschlichkeit und

1 Vgl. Gilroy, Paul: The Black Atlantic. Modernity and Double Consciousness. London: Verso 1993. 
Mitmenschlichkeit ein, die im Grunde nichts von ihrer Bedeutung verloren haben. Seine Brevísima relación de la destrucción de las Indias ${ }^{2}$ bildet fraglos eine der großen Gegenstimmen gegen die Brutalität der spanischen Conquistadoren, die bis heute nicht verhallt ist. Und seine monumentale Historia de las Indias bildet bis heute eine entscheidende Darstellung der Entwicklungen in jener Epoche, die wir als die erste Phase beschleunigter Globalisierung bezeichnet haben.

Der durch seine vehemente Kritik am rücksichtslos plündernden, zerstörenden und massakrierenden Vorgehen der Spanier in Amerika bekannt gewordene Dominikaner behandelte in seiner Geschichte der Eroberung der Neuen Welt ganz selbstverständlich auch die Versuche der Spanier - und früher noch der Portugiesen -, sich des Nordens Afrikas zu bemächtigen und den Schwung der eigenen Reconquista nunmehr für die Eroberung der arabischen 'Gegenküste' zu nutzen. Fray Bartolomé de Las Casas folgt den portugiesischen Geschichtsschreibern seiner Zeit sehr eng, wenn er von den gezielten Erkundigungen der Portugiesen (und insbesondere von Heinrich dem Seefahrer) spricht, die nach der Einnahme von Ceuta, gleichsam der afrikanischen Säule des Herkules, an jeglicher Art von Informationen über die ihnen so unbekannte Welt Afrikas höchst interessiert gewesen seien. So schrieb er diesbezüglich:

Este infante comenzó a tener inclinación de inquirir y preguntar a los moros, con quien allí trataba, de los secretos interiores de la tierra dentro de Africa, y gentes y costumbres que por ella moraban, los cuales le daban relación de la nueva y fama que ellos tenían, que era la tierra extenderse mucho adelante, dilatándose muy lejos hacia dentro de la otra parte del reino de Fez, allende el cual se seguían los desiertos de Africa, donde vivían los alárabes. A los alárabes se continuaban los pueblos de los que se llamaban azenegues, y éstos confinaban con los negros de Jolof, donde se comeinza la región de Guinea, a la cual nombraban los moros Guinauha, del cual tomaron los portugueses y comenzaron a llamar la tierra de los negros, Guinea. [... ..$^{3}$

Man merkt diesen Zeilen des spanischen Dominikaners deutlich an, wie ungewiss man sich in Europa all jener Landschaften und Völkerschaften war, die sich südlich des mittelmeerischen Küstenstreifens in das Innere des afrikanischen Kontinents erstreckten und diesen bevölkerten. Es gab diesbezüglich aber lange Zeit keine verlässlichen Quellen und Aussagen. Dies änderte sich in der ersten Hälfte des 16. Jahrhunderts.

2 Vgl. die deutschsprachige Ausgabe von Las Casas, Bartolomé de: Kurzgefaßter Bericht von der Verwüstung der Westindischen Länder. Übersetzung von D.W. Andreae. 1709 in Berlin erstmals erschienen. Herausgegeben von Hans Magnus Enzensberger. Frankfurt am Main: Insel Verlag 1981.

3 Las Casas, Fray Bartolomé de: Historia de las Indias. Edición de Agustín Millares Carlo y estudio preliminar de Lewis Hanke. 3 Bde. México: Fondo de Cultura Económica 1986, Bd. 1, S. 119. 
Denn über all diese (wohlgemerkt: nur den Europäern) unbekannten Gebiete - insbesondere des Inneren Afrikas - gab die von Giovan Battista Ramusio 1550 herausgegebene (und bearbeitete) Beschreibung Afrikas ausführliche Kunde, ein Werk, das noch bis zu den Zeiten von Mungo Park das eigentliche Standardwerk in Europa über den afrikanischen Kontinent darstellen sollte. Ihr Verfasser war kein Anderer als der wohl zwischen Dezember 1494 und August 1495, also kurz nach ${ }^{4}$ der 1492 erfolgten Eroberung der Hauptstadt des Nasridenreiches im maurischen Granada geborene al-Hassan ben Mohammed ben Ahmed al-Wazzan al-Gharnati al-Fassi, der unter dem Namen Giovan Leone Affricano oder auch Leo Africanus in die Geschichtsbücher und Enzyklopädien der Welt einging. Wer aber war dieser gelehrte Verfasser jener bedeutenden Schrift über Afrika? Und woher bezog er seine Informationen über den 'unbekannten' Kontinent?

Der volle arabische Name des Granadiners enthält nicht nur die erkennbaren Hinweise auf verschiedene Orte, an denen der junge Mann gelebt hatte, sondern wird auch ergänzt durch den christlichen Taufnamen, den der kunstsinnige Papst Leo X. am 6. Januar 1520, also just am Jahrestag der Einnahme Granadas durch die Katholischen Könige, dem späteren Verfasser der Descrittione dell'Africa verlieh. Kein Wunder also, dass der Mann mit den vielen Namen schon früh als ein Wanderer zwischen den Welten oder als Nomade zwischen den Kulturen bezeichnet wurde. ${ }^{5}$ Denn sein Lebenslauf führte ihn von der nördlichen zur südlichen Seite des Mittelmeeres, um ihn zunächst für längere Zeit wieder in den Norden zu führen, bevor er sich endgültig gen Süden aufmachte und für immer verschwand. Mit diesem Wechsel waren natürlich auch die Verortungen in einer islamisch beziehungsweise christlich geprägten Welt verbunden, also jenen sich einander schroff gegenüberstehenden Mächten, die sich bis ins 20. Jahrhundert hinein die Küsten des Mittelmeeres streitig machten.

Zweifellos zählt der in Granada Geborene zu den faszinierendsten Gestalten des frühen 16. Jahrhunderts und kann als eine Art Mittlerfigur zwischen der islamischen und der christlichen Welt rund um das Mittelmeer verstanden

\footnotetext{
4 Allerdings geht al-Wazzans französischer Herausgeber und Übersetzer Alexis Epaulard von einer Geburt möglicherweise um 1489 aus. Vgl. hierzu Rauchenberger, Dietrich: Johannes Leo der Afrikaner. Seine Beschreibung des Raumes zwischen Nil und Niger nach dem Urtext. (Orientalia biblica et christiana, 13). Wiesbaden: Harrassowitz 1999, S. 11 und 35; sowie Redouane, Najib: Histoire et fiction dans 'Léon l'Africain' d'Amin Maalouf. In: Présence francophone (Sherbrooke, Québec) 53 (1999), S. 78.

5 Auf eine lange Tradition zurückgehend findet sich diese Formulierung neuerdings schon im Titel von Davis, Natalie Zemon: Trickster Travels. A Sixteenth-Century Muslim between Worlds. New York: Hill and Wang 2006.
} 


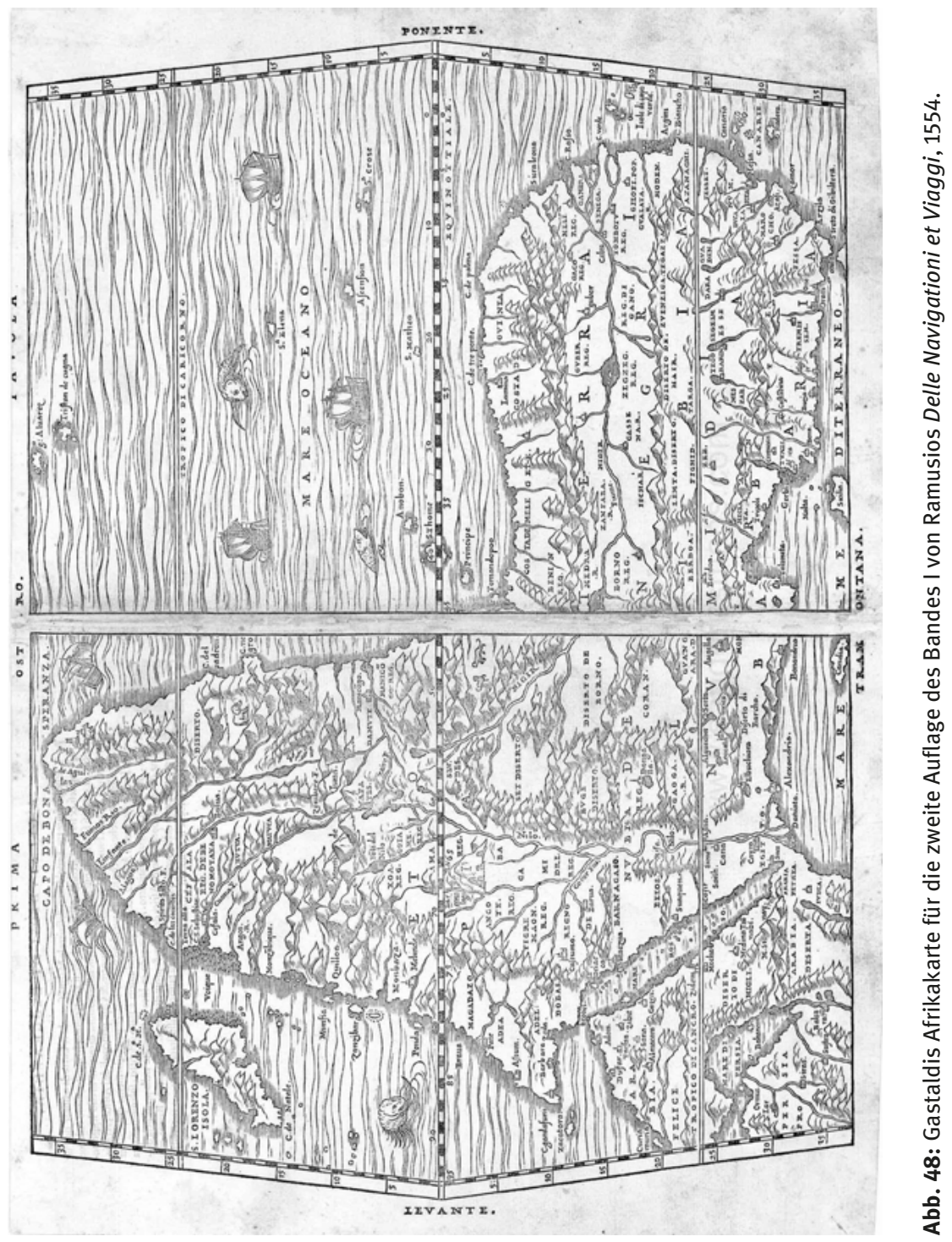




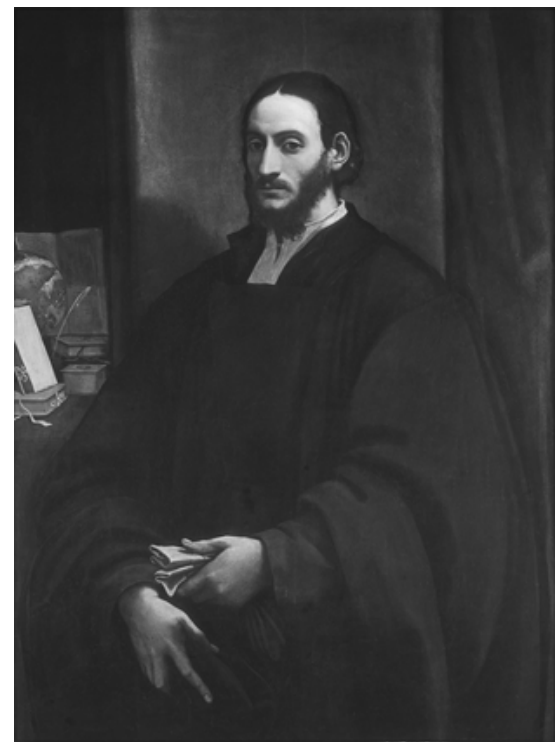

Abb. 49: Johannes Leo Africanus alias al-Ḥasan b. Muḥammed al-Wazzān al-Fāsī (Granada, ca. 1490 - Tunis, 1550).

werden. Mit guten Gründen nahm Giovan Battista Ramusio das ihm wohl in Venedig zugänglich gewordene Manuskript des nach dem Namen des kunstverliebten Medici-Papstes getauften arabischen Reisenden in seine so gewichtige, die globalen Entwicklungen seiner Zeit repräsentierende Sammlung auf. Denn Ramusios Delle navigationi et viaggi wurde zum Standardwerk der Renaissance über die Kenntnisse wie die Ausbreitung der Europäer über den gesamten Planeten.

Zahlreich sind die großartigen Beschreibungen von Städten, die der Granadiner auf seinen Reisen besuchte und deren Bilder er in seinem Reisebericht festhielt. Wir verfügen kaum über lebendigere, anschaulichere Darstellungen der Städte Afrikas als jene, die in seinen reiseliterarisch gekonnt ausgeführten Berichten überliefert sind. Ramusio hatte allen Grund, Leo Africanus in den von ihm ausgewählten Kreis von reiseliterarischen Gewährsleuten und damit in den ersten Band seiner Sammlung aufzunehmen.

Al-Hassan al-Wazzans Beschreibung der Stadt Kairo, die er aus längeren Aufenthalten kannte, ist gleichzeitig auf einen zumindest doppelten, okzidentalen und orientalen, Leserkreis zugeschnitten, beginnt - wie häufig bei diesem Autor - mit Ausführungen zur sprachlichen Herkunft des Namens und schildert die Pracht ihrer Anlage wie die Fülle der in ihren Mauern versammelten Waren und Luxusgüter mit beredten Worten: 
Von Kairo, das gerüchteweise überall für eine der größten und bewundernswürdigsten Städte in der Welt bezeichnet wird, will ich Gestalt und Einrichtung nacheinander beschreiben und die Unwahrheiten, die man darüber hier und da erzählt, übergehen. [. . .] Ich behaupte, daß Kairo, der mit Mauern umgebene Teil nämlich, ungefähr 8000 Feuerstellen enthält. In diesem wohnen die Personen der höheren Stände, und hier werden die von allen Seiten herbeigebrachten Kostbarkeiten verkauft. [...] Die Stadt ist mit Handwerkern und Kaufleuten aller Art reichlich versehen. Das gilt besonders von der ganzen Straße, die vom Siegestor zum Tor Zuwaila führt, wo sich die meisten und vornehmsten aufhalten. In derselben Straße sind einige Kollegien, die wegen ihrer Größe und Schönheit, wegen der Bauart und Verzierungen bewundernswert sind, desgleichen sehr viele und große Moscheen. [. . . ] Hernach folgen die Tuchgewölbe, deren jedes unzählige Läden enthält. Im ersten verkauft man ausländische Tuche von ausnehmender Güte, z. B. Baalbekische, das sind Baumwollstoffe von unglaublicher Feinheit, auch andere, die man nach Mossul benennt (= Musselin). Sie sind bewundernswert fein und fest; daraus lassen alle vornehmen Herren und angesehenen Personen ihre Hemden und die Turbantücher machen. Weiterhin stehen die Gewölbe, wo die besten italienischen Stoffe zu kaufen sind, z. B. Atlas, Damast, Samt, Taft, Brokat. Ferner gibt es die Gewölbe mit Wolltuch, das aus Europa, zum Beispiel aus Venedig, Florenz, Flandern und allen anderen Ländern, kommt.

Nahe bei dieser Hauptstraße ist ein Gewölbe, wo die persischen Kaufleute logieren. Es sieht aus wie der Palast eines großen Herrn, ist sehr hoch und fest und hat acht Stockwerke; unten sind Zimmer, wo die Kaufleute Besuch empfangen und die Waren en gros vertauschen. Nur die allerreichsten Kaufleute handeln hier, und ihre Waren sind Spezereien, Juwelen, indische Stoffe, z. B. Flor und dergleichen. Auf der anderen Seite der Straße ist der Platz für jene, die mit Parfümerien handeln, z. B. Zibet, Moschus, Ambra und Benzoe. [...]. Die Goldschmiede sind Juden und verkaufen viele Kostbarkeiten. ${ }^{6}$

Dies ist ein Bild der Alten Welt, jener Alten Welt, in der Kairo nahe des östlichen Mittelmeeres noch die große Handelsstadt war, in der die Karawanen aus dem Orient, welche die Reichtümer Indiens und Chinas herbeischafften, sich trafen mit den Seehandelswegen der Europäer, insbesondere der Venezianer, die etwa europäische Tuche und Spezialitäten feilboten. Es ist, als hätte sich nichts verändert, so als durchliefen noch immer die traditionellen Karawanenstraßen, ja die alte Seidenstraße, noch immer die asiatische Welt, um sich an dieser Stelle mit den Handelswegen Europas zu kreuzen. Und doch sollte diese Handelswelt mit ihren Kaufleuten bald vor dem Kollaps stehen.

Johannes Leo Africanus alias al-Hassan al-Wazzan hat mit eigenen Augen den Reichtum Kairos gesehen, aber auch den Untergang der Stadt am Nil bei der Eroberung durch die türkischen Truppen von Sultan Selim selbst miterlebt. In seinem Reisebericht, in seiner Erinnerung ersteht die ganze (hier nur ausschnitthaft wiederzugebende) Fülle einer Welt transkontinentalen Handels wieder auf,

6 Johannes Leo Africanus: Beschreibung Afrikas. Herausgegeben von Karl Schubarth-Engelschall. Leipzig: VEB F.A. Brockhaus Verlag 1984, S. 218-221. 
deren zum Teil Jahrtausende alte Handelswege sich in Knotenpunkten wie Kairo, Fez oder Konstantinopel kreuzten. Noch hat sich die Umleitung der Fernhandelswege zwischen Europa und Indien oder China nicht bemerkbar gemacht, noch hat vor allem der Reichtum, der aus den tropischen Ländern des amerikanischen Kontinents zunehmend nach Europa schwappte, nicht zu einer grundlegenden Infragestellung der alten Karawanenwege geführt. Leo Africanus erfasst hier eine Welt kurz vor ihrer Krise; und es gelingt ihm, alle Details dieser Welt präzise und detailgetreu wiederzugeben.

Mit großer Systematik und einer beeindruckenden Fülle an genauen Beobachtungen entwirft der Granadiner Schriftsteller das Weltbewusstsein einer Alten Welt zu einem Zeitpunkt, als die neuen Seewege und Seemächte im Westen längst neue Spielregeln und neue Machtpole zu schaffen im Begriff standen. Die Welt, die Giovan Leone beschreibt, ist nicht nur wegen der immer erdrückender werdenden türkischen Vormachtstellung im östlichen Mittelmeer eine Welt, die es in dieser Form schon bald nicht mehr geben sollte. So ist sein Zeugnis für uns doppelt wertvoll, zeigt es uns doch prächtige Länder, die wir mehr den Erzählungen von Scheherazade und der orientalischen Opulenz von Tausendundeiner Nacht entsprungen glaubten.

Mit diesem kenntnisreichen Gemälde von Kairo wird anhand der Waren und ihrer Herkunftsorte im Überschneidungsbereich von Orient und Okzident, von Afrika, Asien und Europa ein Raum beschworen, in dem die verschiedenen altweltlichen Völker und Kulturen friedlich nebeneinander und miteinander also multi- und interkulturell - zu verkehren scheinen. Innerhalb dieser reiseliterarisch entworfenen altweltlichen Diegese wird eine Welt evoziert, von deren Gewalt der mit seiner Familie aus Granada Vertriebene freilich sehr wohl wusste, die er aber nicht nur im transmediterranen Bereich des Spannungsfeldes zwischen Orient und Okzident, sondern auch in jenen weit entfernten Gebieten im Inneren Afrikas mehrfach durchquert hatte. Denn Leo Africanus war ein weitgereister Mann, mit dem sich nicht viele vergleichen konnten; doch zu seinen vielen Bewegungen im Raume zählten auch erzwungene Reisen.

Er kannte aus eigener Erfahrung jenen Binnenraum des afrikanischen Kontinents, von dem Portugiesen und Spanier wie auch das gesamte christliche Europa nur schemenhafte Vorstellungen besaßen, wie kaum ein anderer arabischer Reisender. Denn schon früh hatte er die alten Handelswege quer durch die Sahara kennengelernt und von jenem Handel profitiert, der sich zwischen den Ländern nördlich und südlich der afrikanischen Wüste etabliert hatte. Timbuktu war für ihn kein ferner Ort mit einem magischen Klang, von dem er nur gehört hätte, sondern konkrete Erfahrung im Sinne all jener Kaufleute, die sich auf diesen Karawanenstraßen bewegten. Sieht man von all jenen Seefahrern, Mönchen, Beamten, Bischöfen, Soldaten und Kaufleuten einmal ab, die während dieses Zeitraums in 


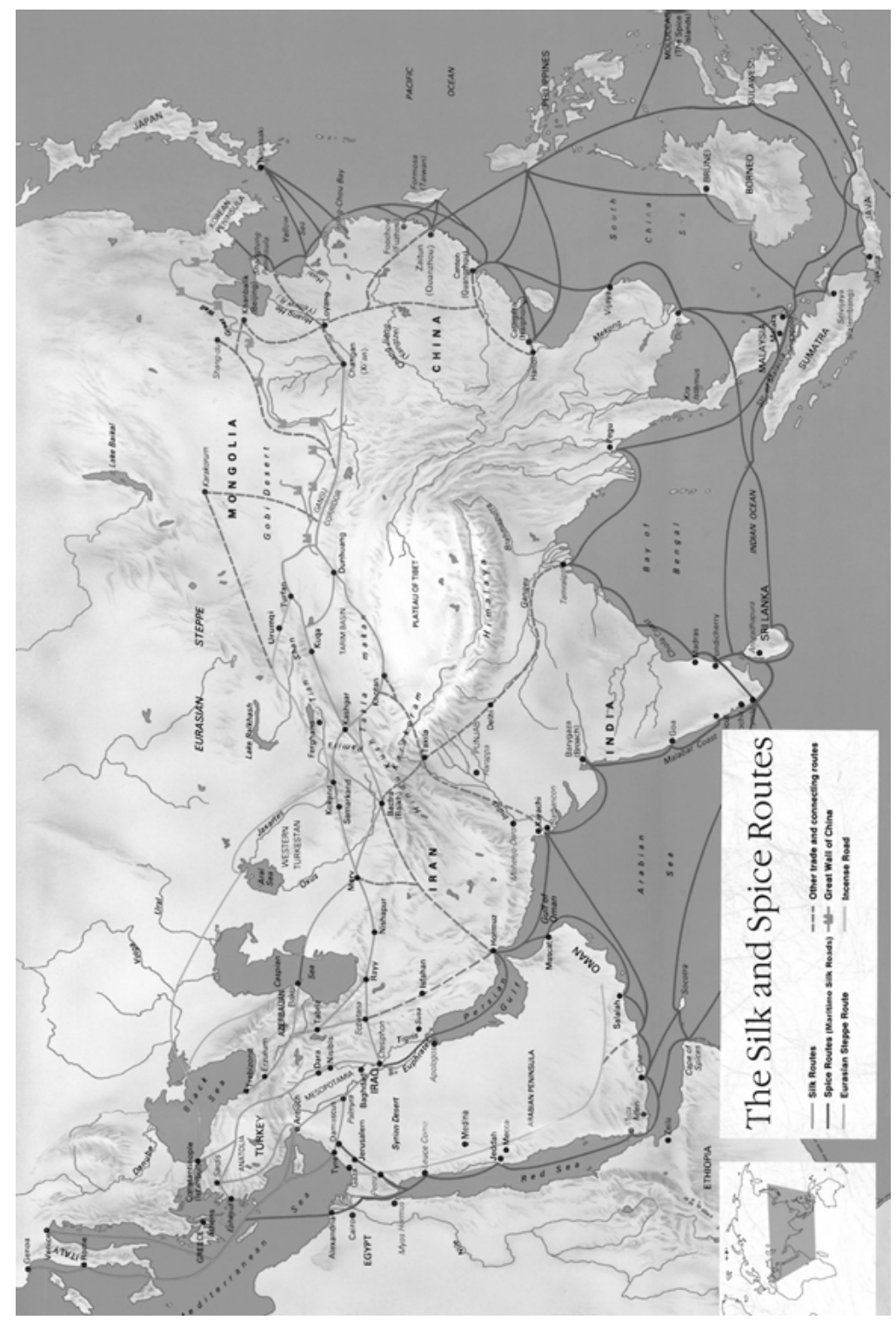

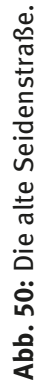


die Neue Welt gelangten, dürften nur wenige Reisende des frühen 16. Jahrhunderts den Erfahrungs- und Kenntnisstand des so weltläufigen Johannes Leo Africanus erreicht haben. Was aber wissen wir über diesen Mann und die Genese seiner Schriften? Was über seine vielen Reisen und die Berichte darüber?

Fassen wir an dieser Stelle die Forschungslage kurz zusammen. Die Forschungen über Giovan Leone L’Africano wurden lange Zeit von französischen Wissenschaftlern und Autoren beherrscht, und dies nicht nur, weil bereits 1553, also nur drei Jahre nach dem Erstdruck der Beschreibung Afrikas durch Ramusio, eine erste französische Übersetzung vorlag. ${ }^{7}$ Die Arbeiten der französischen Forscher zielten zu Beginn vor allem auf die positiven Fakten, auf ein möglichst genaues Wissen hinsichtlich des so schillernden, aber schwer fassbaren Protagonisten. Studien wie die im Kontext der französischen Kolonialpolitik entstandene Doktorarbeit von Louis Massignon ${ }^{8}$ oder die editorischen Arbeiten und Übersetzungen von Alexis Epaulard ${ }^{9}$ hatten $\mathrm{zu}$ einem verbesserten Kenntnisstand rund um die von zahlreichen Legenden umrankte Gestalt des granadinischen Reisenden beigetragen. Eine Neuauflage der von Epaulard besorgten Übersetzung der Description de l'Afrique des Gelehrten erschien im Jahre 1980, fast zeitgleich übrigens mit einer im selben Jahr vorgelegten Übersetzung ins Arabische. ${ }^{10}$ Das Interesse an Leo Africanus und seiner Perspektive auf die erste Phase beschleunigter Globalisierung wuchs just in jener Zeit, in welcher die vierte Phase beschleunigter Globalisierung an Fahrt aufnahm.

Auch zur Textgenese liegen wichtige Erkenntnisse vor. Geht man der Geschichte der Ausgaben der Descrittione dell'Africa nach, so darf man nach dem aktuellen Forschungsstand davon überzeugt sein, dass der von Giovanni Battista Ramusio bekannt gemachte Text von al-Wazzan auf Italienisch zwischen 1524 und 1526 verfasst und abgeschlossen wurde. Also ein Vierteljahrhundert vor seiner ersten Veröffentlichung und Bekanntmachung. Nicht allein der Veröffentlichungskontext in Ramusios angesehener Sammlung der fünfziger Jahre, sondern auch der Entstehungskontext der Beschreibung Afrikas ein Vierteljahrhundert zuvor legt den unmittelbaren Zusammenhang der verschiedenen Reisen wie der Schriften des Johannes Leo Africanus mit der

7 Vgl. hierzu Rauchenberger, Dietrich: Johannes Leo der Afrikaner, S. 153. Der erste Band von Ramusios Sammlung war mit dem Bericht des Leo Africanus im Jahre 1550 erschienen.

8 Vgl. Massignon, Louis: Le Maroc dans les premières années du XVIe siècle. Tableau géographique d'après Léon l'Africain. Alger: Typographie Adolphe Jourdan 1906.

9 Vgl. Jean-Léon l'Africain: Description de l'Afrique. Traduit de l'Italien par Alexis Epaulard et annoté par Alexis Epaulard, Théodore Monod, Henri Lhote et Raymond Mauny. Paris: Librairie d'Amérique et d'Orient 1956.

10 Vgl. Rauchenberger, Dietrich: Johannes Leo der Afrikaner, S. 155. 
ersten Phase beschleunigter Globalisierung nahe. Mehr noch: Sein Bericht ist ein Bestandteil dieser Welle abendländischer Expansion, von deren Macht und Ausbreitung er direkt oder indirekt Zeugnis gibt. Nicht zuletzt auch die Erstveröffentlichung durch Ramusio belegt, dass bereits die Zeitgenossen die Descrittione dell'Africa unmittelbar mit jener Ausweitung der europäischen Kenntnisse über die Welt in Verbindung brachten, die am Ausgang dieser Phase in der Kollektion des gelehrten Italieners noch einmal festgehalten wurde. Ramusios wichtiges Werk Delle navigationi et viaggi zeigt, wie diese erste Phase als ein recht kompakter Zeit- und Erfahrungsblock Mitte des 16. Jahrhunderts verstanden wurde.

Es war historisch gesehen gewiss auch kein Zufall, dass sich im weiteren Verlauf der 1980er Jahre, als die erneute Beschleunigungsphase einer zunehmend alle Bereiche erfassenden Globalisierung fühlbar wurde, sich gerade auch im Bereich der Literatur ein erneuertes Interesse an der Figur des großen Granadiners und Wanderers zwischen den Welten bemerkbar machte. Denn die Literatur ist ohne jeden Zweifel ein Seismograph von (auch historischen) Entwicklungen, die sich erst sehr viel später in ihrer Deutlichkeit abzuzeichnen pflegen. Seit dem Jahre 1986, dem Erscheinen des überaus gelungenen und erfolgreichen Romanerstlings Léon l'Africain des im Libanon geborenen Schriftstellers Amin Maalouf, ${ }^{11}$ lässt sich jedenfalls eine weitaus umfänglichere und tiefergehende Auseinandersetzung mit dem maurischen Andalusier feststellen. Maaloufs Erfolgsroman hatte daran zweifellos großen Anteil. Denn er erreichte ein sehr breites, aber zugleich auch ein spezialisiertes internationales Lesepublikum.

Als die wohl besten Beispiele erwähnt seien hier die 1991 erschienene Arbeit der Marokkanerin Oumelbanine Zhiri, ${ }^{12}$ die die jahrhundertelange Wirkung der gedruckten Schriften al-Wazzans auf das europäische Afrikabild untersuchte, das kulturtheoretisch interessante, aber durch manche handwerkliche Fehler beeinträchtigte 2006 erschienene Buch der in Princeton arbeitenden Forscherin Natalie Zemon Davis ${ }^{13}$ sowie vor allem die 1999 vorgelegte umfangreiche biographische und editorische Untersuchung des Berufsoffiziers und Diplomaten Dietrich Rauchenberger. ${ }^{14}$ Und dies sind nur drei - freilich besonders wichtige Beispiele für die Fülle an Studien, die nun zu erscheinen begann.

11 Vgl. hierzu das dritte Kapitel 'Positionen' in Ette, Ottmar: ZusammenLebensWissen. List, Last und Lust literarischer Konvivenz im globalen Maßstab (2010).

12 Zhiri, Oumelbanine: L'Afrique au miroir de l'Europe: Fortunes de Jean Léon l'Africain à la Renaissance. Genève: Librairie Droz 1991; vgl. auch dies.: Les sillages de Jean Léon l'Africain: XVIe au XXe siècle. Casablanca: Wallada 1995.

13 Vgl. Davis, Natalie Zemon: Trickster Travels. A Sixteenth-Century Muslim between Worlds. 14 Vgl. Rauchenberger, Dietrich: Johannes Leo der Afrikaner. 
Gerade die gegenüber Davis vielleicht etwas spröden Forschungen Dietrich Rauchenbergers haben uns eine sehr viel präzisere Kenntnis der Reisen al-Hassan al-Wazzans ins Innere Afrikas vermittelt. Dabei wird nicht nur anschaulich in einer imaginären Karte vor Augen geführt, wie sich Johannes Leo Africanus den afrikanischen Kontinent und dessen Umrisse zum Zeitpunkt der Niederschrift seiner Beschreibung vorstellte, ${ }^{15}$ in einer itinerarischen Übersichtskarte wurden auch die bislang bekannten oder direkt erschließbaren Verläufe der weiten Reisen im Sahararaum zusammengestellt, so dass erkennbar wird, welch ungeheure Fläche des Kontinents sich der vielsprachige Schriftsteller zu Fuß wie auf dem Rücken von Kamelen erschloss. Zurecht darf er als ein ausgesprochener Afrikakenner gelten, der sich in zahlreichen Reiseverläufen eine intime Kenntnis des freilich unüberschaubaren Kontinents zugänglich machte. Doch noch war aus ihm nicht der Schriftsteller Johannes Leo Africanus geworden.

Die erste seiner ausgedehnteren Reisen führte al-Hassan in den Jahren 1507 und 1508 allerdings nach Konstantinopel, nach Mesopotamien, Armenien, Persien und in die Tartarei. ${ }^{16}$ Er wusste also, wovon er sprach, wenn er die unterschiedlichsten Reichtümer des Orients genauestens beschrieb und wie ein Kaufmann gemäß ihrer jeweiligen Vorzüge festhielt. Seine zweite längere Reise unternahm er dann gemeinsam mit seinem Onkel um 1510 quer durch die Sahara nach Timbuktu, wohin sein Verwandter vom marokkanischen Sultan in diplomatischer Mission gesandt worden war. Hier stand er nun erstmals vor den Landschaften und Städten des afrikanischen Kontinents und begann, ein Gespür für das Innere Afrikas zu entwickeln.

Die dritte Reise (vgl. Abb. 52: „Johannes Leo der Afrikaner: Reisen im Sahararaum“ nach Dietrich Rauchenberger), wahrscheinlich zwischen 1512 und 1514, brachte ihn zunächst gleichfalls transsaharisch nach Timbuktu, verlief dann aber durch die Haussa-Staaten und das Tschadsee-Gebiet weiter in Richtung Osten bis hinauf nach Ägypten. Die vierte und letzte seiner großen Reisen führte al-Hassan al-Wazzan schließlich im Alter von höchstens fünfundzwanzig Jahren abermals in den Norden Afrikas und jenes Gebiet, das wir heute aus eurozentrischer Sicht gerne als den Nahen Osten bezeichnen. Hier erweiterte er wesentlich seinen Horizont innerhalb des arabischen Kulturraumes und begriff, wie vielfältig die Kulturen waren, denen er sich selbst zurechnen durfte.

Von Ägypten aus schloss der gläubige Muslim eine Pilgerfahrt nach Mekka an und begab sich danach wieder auf die Heimreise gen Westen. Doch

15 Ebda., S. 195.

16 Schubarth-Engelschall, Karl: Leo Africanus und seine 'Beschreibung Afrikas'. In: Johannes Leo Africanus: Beschreibung Afrikas, S. 7-18. 


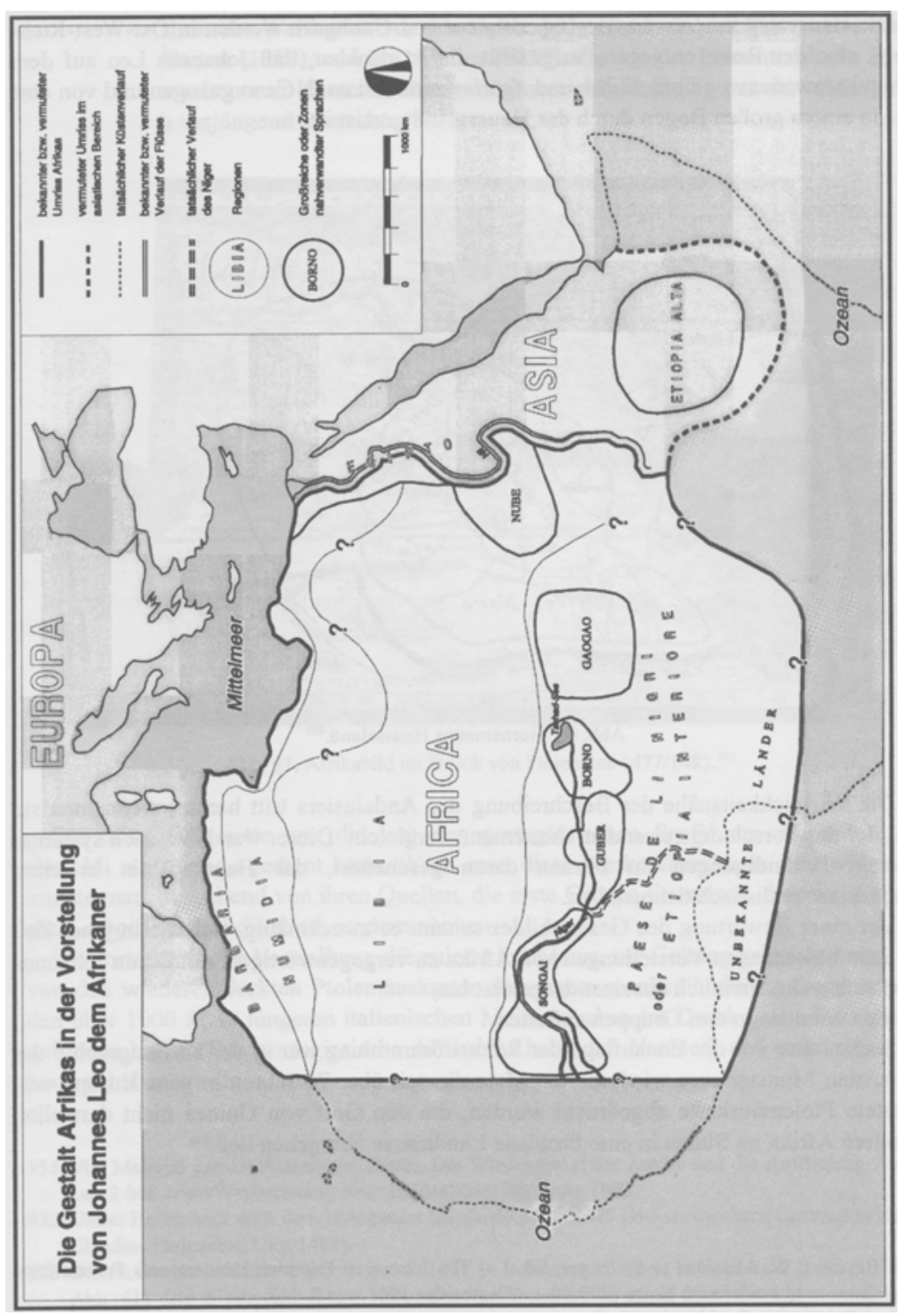

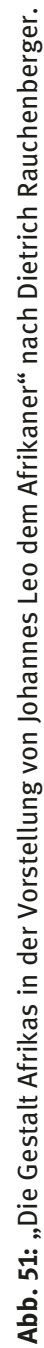




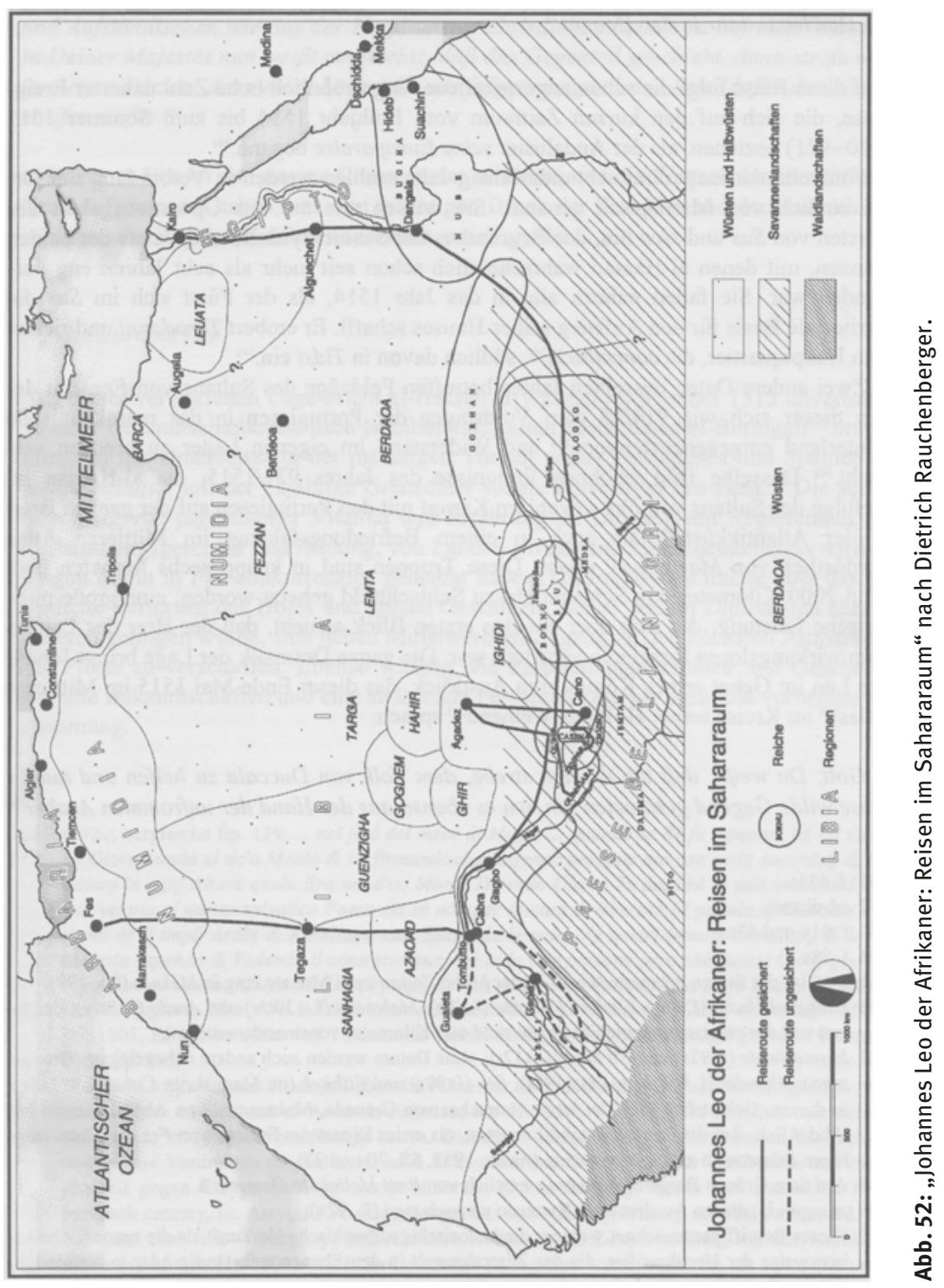


hier begannen jene unliebsamen Überraschungen, die ihn wieder von der Südseite des Mittelmeeres auf dessen Nordseite führen würden: Er sollte sein Fez nicht mehr erreichen. Wir haben im Kontext der Entwicklung von Christoph Columbus bereits von Korsaren im Mittelmeer gehört: Nun haben sie ihren zweiten Auftritt in unserer Vorlesung.

Denn vermutlich während eines Abstechers auf die Mittelmeerinsel Djerba wurde er von christlichen Korsaren unter der Führung von Pedro de Bobadilla gefangen genommen und im Jahre 1518 als Sklave nach Italien verschleppt. Dort wurde er Papst Leo X. zum - für die Zeit keineswegs unüblichen - lebendigen Geschenk gemacht. So kam der weitgereiste und gelehrte Moslem ins Zentrum des christlichen Unglaubens, an den Heiligen Stuhl zu Rom. Aus heutiger Sicht lässt sich sagen: Dies war der Einschnitt, der den Granadiner zum Afrikaner machte.

Sein päpstliches Gegenüber war kein anderer als der berühmte Leo X., der als Freund und Förderer der Wissenschaften und der Künste auftrat und als der große Renaissance-Papst gegen Martin Luther den Kirchenbann verhängte sowie den bekannten Ketzerprozess anstrengte. Dem jungen, gebildeten Muslim al-Hassan al-Wazzan aber begegnete dieser Medici-Papst sehr offen und freisinnig. Er erkannte in ihm einen in weltlichen wie in religiösen Dingen gebildeten jungen Gelehrten, dem er alle Möglichkeiten der Weiterbildung in seinem noblen Gefängnis, der Engelsburg, gerne einräumte, um sich wenn möglich seines Wissens zu versichern und ihn zum Christenmenschen zu konvertieren. Papst Leo X. erblickte darin auch die Chance, die Attraktivität wie die Macht der Katholischen Kirche unverkennbar und weithin sichtbar unter Beweis zu stellen.

Die Interessenlagen waren auf beiden Seiten klar. Aus vielen diplomatischen und anderen Zeugnissen wissen wir, dass die 'Bekehrung' al-Hassan al-Wazzans, des Anhängers des Propheten Mohammed, und dessen Taufe am Heiligen Stuhl zu Giovan Leone ein in vielen Teilen der Christenheit diskutiertes internationales Ereignis war, das ganz im Zeichen des spannungsgeladenen Kräftemessens zwischen Orient und Okzident stand. Papst Leo X. konnte mit dieser Konversion folglich einen Achtungserfolg und ein deutlich sichtbares Zeichen verbuchen - und hatte zudem für die Sache der Christenheit einen aussagekräftigen Informanten gewonnen. Denn längst hatte der illustre Konvertit ein nachhaltiges Interesse an der Bibliothek am Heiligen Stuhl und den Studien zwischen Orient und Okzident gewonnen.

Es ging letztlich um die Frage der Zirkulation des Wissens zwischen Morgenland und Abendland und zugleich um die Frage, wer von einem 'Anzapfen' der Zirkulationsströme am meisten profitieren würde. In dieser Spielart des Ost-West -Konflikts war durchaus ein nachhaltiges Interesse am (Wissen des) Anderen 
vorhanden. So rückte der junge Mann aus Granada, der sich nun wieder auf der nördlichen Seite des Mittelmeeres wiederfand, sehr rasch in eine Vermittler- und Übersetzerposition zwischen der abendländischen und der morgenländischen Welt ein. Als Johannes Leo Africanus hatte er jene Rolle gefunden, für die er berühmt werden sollte. Und er füllte diese Rolle konsequent aus.

Für jegliche Verbindung zwischen Orient und Okzident waren die Frage der Sprachen und deren Übersetzungen von entscheidender Bedeutung. Der folglich mit seinem neuen Taufnamen Johannes Leo ausgestattete Konvertit, der unter dem persönlichen Schutz 'seines' Papstes stand, verfasste unter anderem im Jahre 1524 als Mitarbeiter ein arabisch-hebräisch-lateinisch-spanisches Wörterverzeichnis sowie im Anschluss dann vor allem seine in italienischer Sprache abgefasste Beschreibung Afrikas, die ihm als Gewährsmann eines Wissens über den so nahen und doch so unbekannten Kontinent den Beinamen 'Africanus' eintrug. Sein auf konkreten Reisen und Erfahrungen beruhender Bericht war mit nichts im Abendland vergleichbar, verfügte er doch über einen Kenntnisstand, der weit jenseits der Möglichkeiten und Reichweiten von Christenmenschen lag.

Johannes Leo Africanus schrieb und diktierte seine Descrizione dell'Affrica e delle cose notabili che quivi sono aus guten Gründen nicht in seiner Muttersprache, sondern in jenem Idiom, in dem man ihn in Rom von Beginn an unterrichtet hatte: Der transkulturellen Dimension seines Lebensweges schließt sich die translinguale Abfassung seines Hauptwerks auf geradezu natürliche Weise an. Und dieses Hauptwerk ist auch noch für ein heutiges Lesepublikum lebendig und zudem in einer Sprache geschrieben, welche längst zur europäischen Trägersprache von Wissen avanciert war. Für den jungen Granadiner, der sich von Haus aus zwischen verschiedenen Sprachen bewegte, war es durchaus selbstverständlich, nicht in seiner eigenen Muttersprache zu schreiben.

Denn mit der Verschiedenheit unterschiedlicher Sprachen war der Granadiner gleichsam von Geburt an sehr vertraut. Als in Granada geborener Muslim war al-Hassan al-Wazzan zweifellos in unterschiedlich engem Kontakt mit dem Arabischen und seinen dialektalen Varianten, mit dem Berberischen, dem Spanischen und den verschiedensten Mischformen zwischen all diesen Sprachen aufgewachsen. Auf seinen ausgedehnten Reisen hatte er eine Fülle afrikanischer Sprachen kennengelernt, bevor er in der Engelsburg im päpstlichen Rom im Lateinischen und Italienischen unterrichtet wurde und in diesen 'abendländischen’ Sprachen (wohlgemerkt: unter Aufsicht und Beobachtung!) Bücher und Schriften zu lesen begann. Als polyglotter Gelehrter und Leser war er daher höchst sensibilisiert für alle Formen inter- und translingualer Sprachphänomene, ebenso für asymmetrische Sprachkontakte wie für unterschiedlichste Übersetzungsproblematiken zwischen jenen Sprachen, die er am besten beherrschte. Auch in dieser Hinsicht bildete die große Sprachenvielfalt rund um das 
Mittelmeer eine entscheidende Voraussetzung für jene Zirkulation des Wissens, die es ins Werk zu setzen galt. Ihm scheint rasch klar geworden zu sein, daß eben hierin seine Chance lag.

Die sprachliche Aufmerksamkeit des jungen Mannes war daher geschärft: Ständige Sprachreflexionen durchziehen folglich sein gesamtes sprachsensibles Schaffen. So geht es in seiner Descrizione dell'Africa nicht nur um die geographischen und topographischen Grenzen Afrikas, um dessen Klima und Boden, Vegetation und Anbauprodukte, um die großen Ströme, die für den Kontinent charakteristischen Tiere, um die verschiedenartigen Völker und deren Handelsgüter, sondern auch um differenzierende kulturelle Merkmale, die vor allem in sprachlicher Hinsicht notiert und untersucht werden. Sein Bericht von all seinen Reisen durch die verschiedenen Regionen Afrikas war nicht nur voller handfester Informationen über Handel und Wandel, sondern durchsetzt mit Überlegungen zu kulturellen Differenzen zwischen den verschiedenen Völkern dieser Weltregion.

Einige wenige Beispiele mögen hier genügen. So werde in „allen afrikanischen Landschaften, die sich vom Mittelmeer bis zum Atlas-Gebirge erstrecken, [...] ein verdorbenes Arabisch gesprochen“, wobei nur „im Reich Marokko sowie in Numidien [...] das Berberische weiter verbreitet“ sei. ${ }^{17}$ „Die arabischen Geschichtsschreiber“, so Giovan Leone weiter, behaupteten „nachdrücklich, daß die Afrikaner keine andere Schrift gehabt haben als die mit lateinischen Buchstaben“; sie hätten zwar andere Sprachen gesprochen, hätten sich aber „lateinischer Buchstaben bedient, wie es die Deutschen in Europa tun“. ${ }^{18}$ Und durchaus quellenkritisch merkt er an, dass ausnahmslos alle „Geschichtsbücher über die Afrikaner, die die Araber besitzen“, Übersetzungen aus dem Lateinischen seien, „alte Werke, geschrieben in den Zeiten der Arianer, einige noch früher“. ${ }^{19}$ Es war ihm zweifellos darum zu tun, diese Zirkulationen des Wissens und der Kenntnisse in dieser Aufbruchphase der italienischen und (bald) europäischen Renaissance wieder in Gang zu setzen. Denn ein solches Bemühen passte wunderbar zu jener historischen Phase, die auf eine Ausweitung des Wissens auch jenseits der Grenzen der Antike drängte und wo mit Blick auf überseeische Wissensbereiche gerade Italien mit seinen Cristoforo Colombo, Amerigo Vespucci, Pietro Martire d'Anghiera und so vielen mehr eine besondere Rolle als Wissensplattform zugefallen war. Denn der Drang nach Wissen war in einem enger zusammengewachsenen Europa schlicht enorm.

17 Johannes Leo Africanus: Beschreibung Afrikas, S. 72.

18 Ebda.

19 Ebda. 
Wenn auch das Afrika, das Johannes Leo Africanus seinem zeitgenössischen Lesepublikum präsentiert, im Zeichen einer Fülle steht, welche Landschaften und Flüsse, Flora und Fauna, aber auch Völkerschaften, Kulturen und Sprachen umfasst, so fehlt auch das Element der Falle nicht, sind doch im Norden des Kontinents längst die Symptome der Syphilis aufgetaucht, die in diesen Landstrichen zuvor völlig unbekannt gewesen sei. Auf diese Weise erscheinen in der Descrittione dell'Africa nicht nur die Erkenntnisse über sich ausweitende Welthorizonte, sondern auch all jene Krankheiten, die - wie bereits betont mit der europäischen Globalisierung einhergingen. Die Syphilis als Globalisierungskalamität konnte hier nicht fehlen.

Für die Syphilis wollte niemand verantwortlich sein, stets schob man die Schuld dafür anderen europäischen Völkern in die Schuhe. Dieses Spiel begann schon kurz nach der Jahrhundertwende, als die Krankheit sich vornehmlich in den Ländern rund um das Mittelmeer zu verbreiten begann. Johannes Leo Africanus bildet hier keine grundsätzliche Ausnahme von der europäischen Regel, weist aber - wie wir gleich sehen werden - eine Besonderheit auf.

Mit der Erörterung dieser Leit-Epidemie der ersten Phase beschleunigter Globalisierung treten wir aus einer transarealen Sichtweise, wie wir sie durchaus in Ramusios Sammlung in nuce finden können, ein in die zeitgenössische Diskussion jener sich weltweit verbreitenden Seuche, die außerhalb von Frankreich als morbo gallico, als französische Krankheit, bezeichnet wurde, auch wenn die Portugiesen sie gerne die kastilische, die Schotten wiederum eher die norwegische Krankheit nannten. Giovan Leone L'Africano schloss sich seinerseits eher den in der arabischen Welt kursierenden Gerüchten an und verfocht die These von einer anderen Herkunft:

Die Französische Krankheit ist in der Berberei sehr verbreitet. Nur wenige Einwohner entgehen ihr. Sie verursacht Beulen und Geschwüre. Auf dem Lande und im AtlasGebirge leidet fast niemand daran. Auch bei den Arabern, in Numidien, in Libya und im Land der Schwarzen kennt man das Übel nicht. Ja, man bringt die Erkrankten sogar nach Numidien und Nigritien, weil sie durch die dortige Luft gesund werden. Ich selbst habe einige hundert Personen gesehen, die durch die bloße Luftveränderung, ohne ein anderes Mittel, geheilt worden waren. Die Seuche war ursprünglich in Afrika selbst dem Namen nach unbekannt. Sie wurde von den Juden eingeschleppt, die durch König Ferdinand aus Spanien vertrieben worden waren. Viele von ihnen waren krank, und die wollüstigen Mauren steckten sich bei den Jüdinnen an, die nach Afrika gekommen waren, so daß bald keine Familie in der Berberei von dem Übel verschont blieb. Anfangs wurden die von der Französischen Krankheit Befallenen als leprakrank angesehen, von ihrem Heim vertrieben und gezwungen, mit den Aussätzigen zu leben. Aber als die Zahl der Erkrankten täglich stieg und eine große Menge von Menschen befallen war, begannen die Kranken wieder ihr normales Leben zu führen, und die Vertriebenen kehrten wieder nach Hause zurück. 
Man hält es für zweifelsfrei, daß die Seuche aus Spanien kam, und nennt sie daher die Spanische Krankheit. In Tunis, wo sie einige Zeit sehr gewütet hat, in Ägypten und Syrien heißt sie, wie in Italien, die Französische Krankheit. ${ }^{20}$

Die Reaktions- und Darstellungsweisen von Johannes Leo Africanus auf die Erscheinungsformen der Leit-Epidemie der ersten Phase beschleunigter Globalisierung sind aufschlussreich. Denn sie zeigen, wie kulturbedingt die jeweiligen Thesen und Hypothesen sind, was Herkunft und Einschleppung von Krankheiten in den 'eigenen' Bereich, in diesem Falle nach Nordafrika, angeht. So ist sich der Granadiner, der selbst von den Spaniern aus der Hauptstadt des Nasridenreiches vertrieben wurde, sicher, dass es die ebenfalls vertriebenen Juden waren, welche die Krankheit einschleppten. So werden bestimmte historische Ereignisse ausgewählt, um den Hintergrund für plausible, aber letztlich rein kulturbedingte Thesen abzugeben. Die Frage, woher die den Juden angedichtete Krankheit stammt, stellt sich der Wanderer zwischen den Welten nicht. Auch wird die überseeische Herkunft der Syphilis bei ihm nicht als Möglichkeit in Betracht gezogen. Er kalkuliert allein mit einer Krankheit, die aus Europa selbst gekommen wäre - und dabei leiten unverkennbar judenfeindliche Aspekte alleine seinen Blick.

Ebenso in den Formen der Berichterstattung wie in den sich aus der Verbreitung der Seuche ableitenden (und zumeist transitorischen) Normen des Zusammenlebens lässt sich vieles mit den Formen und Normen in Verbindung bringen, mit denen auch in der zweiten, dritten und vierten Phase beschleunigter Globalisierung auf Epidemien und Pandemien als grundlegenden Globalisierungssymptomen und -ängsten reagiert wurde. Lassen sich hier nicht eindeutige Parallelen zwischen den Reaktionsweisen auf die Verbreitung der Syphilis in der ersten und auf die zunächst unerklärliche Ausweitung der AIDS-Epidemie in der vierten Phase beschleunigter Globalisierung ziehen? Sind hier die Reaktionsweisen im Übergang zwischen Mittelalter und Renaissance einerseits und in einer 'aufgeklärten' postmodernen Gesellschaft andererseits nicht nahezu identisch? Selbst in Ländern wie Schweden oder Deutschland war eine strenge Kasernierung von Erkrankten angedacht und 'Lösungen' des Problems im Gespräch, wie sie letztlich mit großer Konsequenz dann im autoritären System Cubas in den ersten Jahren umgesetzt wurden.

Insofern liefert uns die Beschreibung Afrikas nicht nur einen spannenden zeitgenössischen Einblick in Problematiken einer Globalisierung, die in der 'Alten Welt' - denken wir nur an die rasch einsetzenden Biopolitiken der Verschiffung schwarzer Sklavinnen und Sklaven in die 'Neue Welt' - gewiss nicht

20 Ebda., S. 75. 
vor dem afrikanischen Kontinent haltmachten. Zugleich zeigt uns dieses Mitte der zwanziger Jahre des 16. Jahrhunderts entstandene Werk mit literarischen Mitteln auf, wie sich in den unterschiedlichsten Bereichen im Zeichen der ersten Phase beschleunigter Globalisierung Lebensformen und Lebensnormen zu verändern begannen und wie die Epidemie sich Stück für Stück 'normalisierte'. Denn die an Syphilis Erkrankten nahmen langsam wieder ihr angestammtes Leben auf und integrierten sich erneut - wie die Beschreibung Afrikas erläutert in ihre jeweiligen Gesellschaften.

Doch die weltpolitischen Rahmenbedingungen und Kontexte hatten sich zu Beginn des 16. Jahrhunderts ein für alle Mal grundlegend verändert. Während am einen Ende der Welt die vorrückenden Türken bald alle terrestrischen Handelsverbindungen zwischen Asien und Europa zu kontrollieren vermochten, errichteten am anderen Ende der Welt die iberischen Mächte ihre Herrschaft auch und gerade über die Länder der Spezereien. Binnen weniger Jahrzehnte war die Welt eine andere geworden: Alte Handelswege wurden langsam immer schwächer, während sich neue Handelswege eröffneten, welche den Schwerpunkt des europäischen Handels (und damit auch der europäischen Politik) an die Westseite des Kontinents verlagerten.

Al-Hassan al-Wassan ist, daran kann kein Zweifel bestehen, eine außerordentliche Schriftstellerpersönlichkeit, die es verstand, aus dem Wandern zwischen den Welten (unabhängig davon, dass es jeweils ein erzwungenes Wandern war) einen inter- und transkulturellen Mehrwert herauszuschlagen, der seine Schriften und Aktivitäten beseelt. Er wurde in seiner Zeit zweifellos zum wichtigsten Mittler zwischen der abendländischen und der morgenländischen Seite des Mittelmeeres.

Man sollte aus diesem Faktum jedoch nicht den irreführenden Schluss ziehen, dadd sein Leben selbst wie auch sein Lebenswissen völlig aus den Rahmenbedingungen seiner Zeit herausgefallen wären. Denn in seiner Zeit der Kriege und Vertreibungen, der Pogrome und Migrationen, der Sklavenjagden und Fluchtwellen, der Hungersnöte und Zwangsaushebungen sind derartige Lebenswege zwischen den Kulturen nicht wirklich singulär. Würden uns aus heutiger Perspektive die Wege Abertausender einfacher Matrosen oder Söldner, Galeerensklaven oder Kaufleute, Marketenderinnen, Dirnen oder Nonnen nicht weniger 'abenteuerlich' erscheinen? Im Falle des Granadiners freilich verbanden sich sein Schicksal und seine Lebenswege mit einer ausßergewöhnlichen Fähigkeit zur Kommunikation in verschiedensten Sprachen und seiner festen Absicht, die Zirkulationen von Wissen zwischen den Welten zu beschleunigen. Im geschriebenen translingualen Wort, im Schreiben jenseits der Muttersprache, fand er schließlich die ihm adäquate Ausdrucksform, mit deren Hilfe er sich einen festen Platz in den Beziehungen zwischen Orient und Okzident 
erschrieb. Wir stoßen hier auf ein Schreiben, das sich deutlich (und erzwungen) nach der Reise anordnet, zugleich aber die eigenen Reisen immer wieder neu in einen sich entwickelnden Zusammenhang des eigenen Schreibens einbettet. Das Verhältnis von eigenem Gereistsein und Schreiben ist bei diesem in Granada geborenen Autor eine hochdynamische Relation.

Johannes Leo Africanus war aus allen diesen Gründen (und vor allem auch schriftstellerisch) fraglos eine beeindruckende historische Persönlichkeit. Weit über seine Zeit hinaus fasziniert an seiner Gestalt jedoch vor allem die Tatsache, dass er in seiner translingualen Tätigkeit als Schriftsteller zwischen Europa, Afrika und Asien eine Form des ZwischenWeltenSchreibens zu entfalten verstand, die wir aus dem Blickwinkel der aktuellen vierten Phase beschleunigter Globalisierung dank der Rekonstruktion der spezifischen historischen Kontexte wohl präziser und sensibler $\mathrm{zu}$ erfassen verstehen als $\mathrm{zu}$ anderen, weniger 'bewegten' und weniger velociferischen Zeiten. Doch vom Hintergrund unserer eigenen Zeiterfahrung aus und im Bewusstsein aktueller Deutungs- und Schreibweisen inter- und transkultureller Begegnungen ist dieser Wanderer zwischen den Welten weitaus sensibler und adäquater zu erfassen. Sein Leben wirkt für uns heutige fast wie ein Roman - und zwar ganz ähnlich jenem Romanerstling, den Amin Maalouf mit Leon l'Africain schuf.

Aus der Not, die Kontinente und die Sprachen, die Kulturen und die Religionen wechseln zu müssen, um überleben zu können, hat al-Hassan al-Wazzan alias Johannes Leo Africanus die transkulturelle Tugend entwickelt, zwischen dem Süden und dem Norden, zwischen dem Osten und dem Westen eine unterschiedliche Kulturen und Ausdrucksformen querende Übersetzungsarbeit zu entwickeln, welche ihn $\mathrm{zu}$ einer der schillerndsten und zukunftsträchtigsten Figuren der ersten Phase beschleunigter Globalisierung machte. Denn seine Descrittione dell'Africa ist weit mehr als eine bloße Beschreibung Afrikas: Sie modelliert mit den Mitteln der Literatur aus einer transarealen Perspektive avant la lettre die Komplexität der alten im Lichte einer heraufziehenden neuen Welt.

Gewiss konnte er noch nicht erkennen, welche fundamentalen Folgen die zunehmende Verlagerung der Welthandelsrouten im Zuge der europäischen 'Entdeckung' Amerikas haben würde. Mit Blick auf den afrikanischen Kontinent begannen sich diese Transformationen erst am Horizont abzuzeichnen und griffen stärker in ein südlich der Sahara liegendes Afrika ein, das bald schon als ideales Jagdgebiet für schwarze Sklaven diente, welche die stetig wachsende Gier nach immer neuen Arbeitskräften auf den europäischen Plantagen der westindischen Inselwelt wie des zirkumkaribischen Raumes befriedigen mussten. Dies entsprach aber noch nicht der Erfahrungswelt des al-Hassan al-Wazzan zum Zeitpunkt seiner langen Reisen über Land. 
$\mathrm{Zu}$ seiner Zeit bestanden die alten Karawanenwege noch immer fort, entspannen sich die Kulturen querenden Beziehungen entlang von Routen, die quer über Land verliefen und wie an einem (seidenen) Faden kulturelle Diversitäten auffädelten. Wenn Spezereien und Gewürze, Gold und Silber aber nun vor allem über die transatlantischen Handelswege transportiert wurden, so sollte dies die gesamten ökonomischen, politischen, sozialen und kulturellen Rahmenbedingungen grundlegend und irreversibel verändern. Was wir von Johannes leo Africanus alias al-Hassan al-Wazzan jedoch noch immer lernen können, das ist die unschätzbare Fähigkeit, verschiedene Kulturen als einander gleichrangig, als äquipollent zu erachten und in unterschiedlichen sprachlichen wie gedanklichen, philosophischen Logiken zugleich zu denken. Er vermochte es, Wissen unterschiedlichster Form in den abendländischen Kontext zu vermitteln, gerade weil er die kulturellen Differenzen kannte und zwischen Orient und Okzident in keiner Weise in Kategorien von Inferiorität und Superiorität dachte. Eben dies macht den im Granada der Katholischen Könige Geborenen so faszinierend auch und gerade für unsere Zeit. 